\title{
Novel Anti- tuberculosis Compound
}

\author{
Prof. Dr. Habeeb Sahib Naher ${ }^{1 *}$, Assist. Prof. Anwar Kadhim AL-Saffar ${ }^{2}$ \\ ${ }^{I}$ Dept. Microbiology, College of Medicine/ Babylon University, Hilla, Iraq) \\ ${ }^{2}$ (Dept. Biology, College of Sciences/ Babylon University, Hilla, Iraq)
}

\begin{abstract}
Mycobacterium tuberculosis, the causative agent of tuberculosis is quiet difficult to treat or say, recovery the patients is prolonged.Iraq has a high burden of Tuberculosis (TB) and ranks the $44^{\text {th }}$. worldwide among the countries with a high TB burden and the $5^{\text {th }}$.among the countries of the Eastern mediterranean Region.This study was designed to evaluate the Anti-tuberculosis effect of a new compound of Argan Oil $100 \%$, which has been fetched from Morocco Kingdom combined with Sidr extract $62.5 \mathrm{~g} \mid \mathrm{L}$ against Mycobacterium tuberculosis.

Ten isolates Mycobacterium tuberculosis were isolated from patients who attended Consultants Clinic of Respiratory and Thoracic Diseases in Hilla city, Iraq. All investigated patients were preliminary diagnosed by $X$ - ray to have TB. The effect of mixture of Argan oil 100\% and sidr extract $62.5 \mathrm{~g} \backslash \mathrm{L}$ was tested on M. tuberculosis by incorporation the mixture with Lowenstein-Jensen (L.J) medium in different ratio to get final volume mixture: L.J. medium. Accordingly ratios were; 1: 9, 0.75:9.25, 0.5: 9.5, 0.4:9.6, 0.3:9.7, 0.25:9.75, 0.2:9.8 and 0.1: 9.9ml. L.J. medium was autoclaved for sterilization and kept to cool at $45 \mathrm{C}^{\circ}$ then the appropriate concentrations the compound were added. The medium was inoculated with the TB.The growth of TBwas observed in positive control tube while no growth was seen in the tubes containing the mixture of compounds although the reading of TB. growth continued for 28 days of incubation. Similar results displayed for all other mixtures of different ratio (1: 9, 0.75:9.25, 0.5: 9.5, 0.4:9.6, 0.3:9.7, 0.25:9.75, 0.2:9.8 and 0.1: 9.9) ml.The new compound used in this study revealed valuable effect against TB. Afterincorporated with culture media.
\end{abstract}

Keywords:Argan oil.Sidr extract.Anti-tuberculosis.

\section{Introduction}

Tuberculosis (TB) is an ancient disease with a long history of co-evolution with humans and a wide geographic distribution. Mycobacteriumtuberculosis, the primary causative agent of TB disease in humans is a member of the M. tuberculosis complex (MTBC), a group of pathogens with high DNA sequence similarity but different host tropism, epidemiology and pathogenicity [1,2].TB currently infects one third of the global population, coming in second after HIV as a cause of death worldwide [3]. One of the most concerning aspects of TB epidemiology is the emergence of multi- and extensively drug resistant strains (MDR and XDR-TB), which cause of concern in many parts of the globe [4].

Iraq has a high burden of tuberculosis (TB) and ranks $44^{\text {th }}$.worldwide among countries with a high TB burden and $5^{\text {th }}$.among the countries of the Eastern Mediterranean Region. According to world health organization, there were 20000 incident cases of TB in Iraq in 2010 [5]. The incidence rate in 2010 was 64 cases per 100000 of population, and the prevalence rate was 117 cases per 100000 of population, based on a total population of 32 million. In 2010, here were 3900 death cases among TB cases, this give a mortality rate of 12 death per 100000 population. The case detection rate was $48 \%$. The estimated multiple drug resistancetuberculosis (MDR-TB) cases among new pulmonarydrug resistance-tuberculosis (MDR-TB) notified in 2010 was 210 (3.4\% of new TB cases) [6]. Emergence of MDR-TB may indicate that the traditional antibiotics already used for treatment of TB become inactive at the present time [7].

Argan oil is a plant oil produced from the kernels of the Argan tree (Arganiaspinosa L.) that is endemic to Morocco. It is used for nutritive and cosmetic purposes. The tree is extremely well adapted to drought and other environmentally harsh conditions of southwestern Morocco. The genus Argania is now endangered and under protection of UNESCO [8]. Argan oil remains one of the rarest oils in the world due to the small and very specific growing areas.

The fruits of the Argan tree are nut-sizes and may be round, oval or conical in shape. The fruits are covered by a thick peel which covers the fleshy pulp. The pulp surrounds a hard-shelled nut which represents approximately $25 \%$ of the weight of the fresh fruit. Argan oil is extracted from the kernels, with yields from $30 \%$ to $55 \%$ depending on the extraction method used [9]. Argan oil consist of $42.8 \%$ oleic, $36.8 \%$ linoleic, $12 \%$ palmitic, $6 \%$ stearic and $<0.5 \%$ Linolenic [10]. Argan oil has a relative density at $20^{\circ} \mathrm{C}\left(68{ }^{\circ} \mathrm{F}\right)$ ranging from 0.906 to 0.919 [11]. Argan oil contains tocopherols (vitamin E), phenols, carotenes, squalene, and fatty acids, ( $80 \%$ unsaturated fatty acids). The main natural phenols in Argan oil are caffeic acid, oleuropein, vanillicacid, 
tyrosol, catechol, resorcinol, epicatechin and catechin[10]. Depending on the extraction method, Argan oil may be more resistant to oxidation than olive oil [12].

Ziziphusspina-christi commonly known as Christ's Thorn Jujube, is a deciduous tree and native to the warm-temperate and subtropical regions, including North Africa, South Europe, Mediterranean, Australia, tropical America, South and East of Asia and Middle East [13]. It belongs to the Rhamnaceae family in the order of Rosales that contains about 60 genera and more than 850 species. The genus Ziziphus consists of about 100 species of deciduous or evergreen trees and shrubs throughout the world [14] .

The Ziziphusspina- Christi was known to be active against wide spectrum of bacteria due to presence of betulic and cyanotic acid, three cyclopeptide alkaloids as well as four saponim glycosides and several flavonoids [15].

\section{Material And Methods}

2.1. Preparation of the Sidrextract: A weight of $62.5 \mathrm{~g}$ of fresh and clean Sider leaves was immersed in one liter of sterile distilled water and heated up to $65 \mathrm{C}^{\circ}$ for overnight. The solution was then left in the refrigerator for 12 hours[16].The solution was filter sterilized, the solid leaves were discarded and an appropriate volume of the liquid content was mixed with concentrated Argan oil ( $100 \%$ ) to get a combined compound of Argan oil $100 \%$-Sider extract $62.5 \mathrm{~g} / \mathrm{L}$. to be used in necessary experiments included in this study.

2.2. Cell Toxicity of the mixture: This experiment was performed as described by [17].

2.3. Preparation of Lowenstein-Jensen (L-J) medium:L-J medium provided by HiMedia Laboratories, India was used and prepared as recommended by [18].

2.4. Bacterial isolates:Ten Mycobacteriumtuberculosis isolates were recovered from patients who attended the consultants clinic of respiratory and thoracic diseases in Hilla city, Iraq. all patients were preliminary diagnosed by clinicians to have TB.

2.5. Experiment:The effect ofcompound was tested on M. tuberculosis by incorporation it with L-.J medium( after autoclaving and cooling to $45 \mathrm{C}^{\circ}$ ) in universal tubes in different ratios of compound $/ \mathrm{ml}$. : L-J medium $/ \mathrm{ml}$. as; $1: 9,0.75: 9.25,0.5: 9.5,0.4: 9.6,0.3: 9.7,0.25: 9.75,0.2: 9.8$ and 0.1: 9.9. The tubes were slant inoculated and incubated for 28 days with following up the growth daily.

\section{Results And Discussion}

The effect of mixture (Argan oil 100\% and sidr extract $62.5 \mathrm{~g} / \mathrm{L}$ ) on TB not studied in Iraq and most of Arabian countries yet.The growth of TB-bacilli was seen in positive control tube, while no growth was seen in tube containing the compound after an incubation of 17 days and even after 28 days. The similar result were obtained for all other mixtures of different ratio $(1: 9,0.75: 9.25,0.5: 9.5,0.4: 9.6,0.3: 9.7,0.25: 9.75,0.2: 9.8$ and $0.1: 9.9) \mathrm{ml}$. The novel compound used in this study revealed valuable inhibitory effect against TB as shown in (Table 1).

Table 1: inhibition effect of mixture in the different ratio after different times

\begin{tabular}{|c|c|c|c|c|c|c|c|c|}
\hline \multirow{2}{*}{$\begin{array}{c}\text { Incubation/ } \\
\text { Days }\end{array}$} & \multicolumn{7}{|c|}{ Ratio of (mixture : L.J. medium) ml } \\
\cline { 2 - 8 } & $\mathbf{1 : 9}$ & $\mathbf{0 . 7 5 : 9 . 2 5}$ & $\mathbf{0 . 5 : 9 . 5}$ & $\mathbf{0 . 4 : 9 . 6}$ & $\mathbf{0 . 3 : 9 . 7}$ & $\mathbf{0 . 2 5 : 9 . 7 5}$ & $\mathbf{0 . 2 : 9 . 8}$ & $\mathbf{0 . 1}: 9.9$ \\
\hline 17 & - & - & - & - & - & - & - & - \\
\hline 21 & - & - & - & - & - & - & - & - \\
\hline 28 & - & - & - & - & - & - & - & - \\
\hline
\end{tabular}

In this study there was growth of the TB -B-bacilli in the media containing Argan oil $100 \%$ only and also growth in media containing sidr extract $62.5 \mathrm{~g} \backslash \mathrm{L}$ while no growth of the TB in the mixture tube in the different ratio $(1: 9,0.75: 9.25,0.5: 9.5,0.4: 9.6,0.3: 9.7,0.25: 9.75,0.2: 9.8$ and 0.1:9.9)ml shown in ( Figure :1 A,B,C,D,E,F,G,H).

The results Regarding the Anti-tuberculosis effect of Argan oil indicated that there was no any considerable effect for Argan oil only and Sidr extract only as well compared with the control, while when these two substances combined with each other properly, a potential compounds can be obtained. 

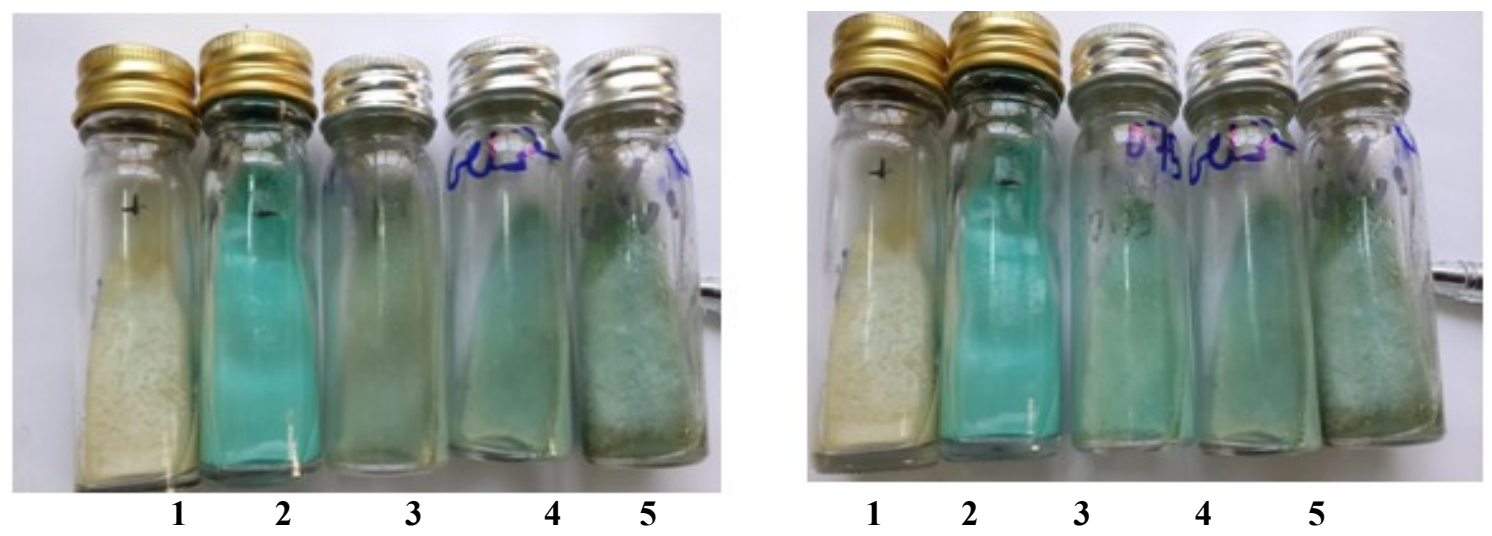

A:B:

1.Positive control tube.1. Positive control tube.

2.Ngative control tube.2. Negative control tube.

3.L-Jwith the compound (9:1) ml.3.L-Jwith the compound $(9.25: 0.75) \mathrm{ml}$

4.L-J with Sidr extract only.

4.L-J with sidr extract only.

5.L-J with Argan oil only. 5.L-J with Argan oil only.
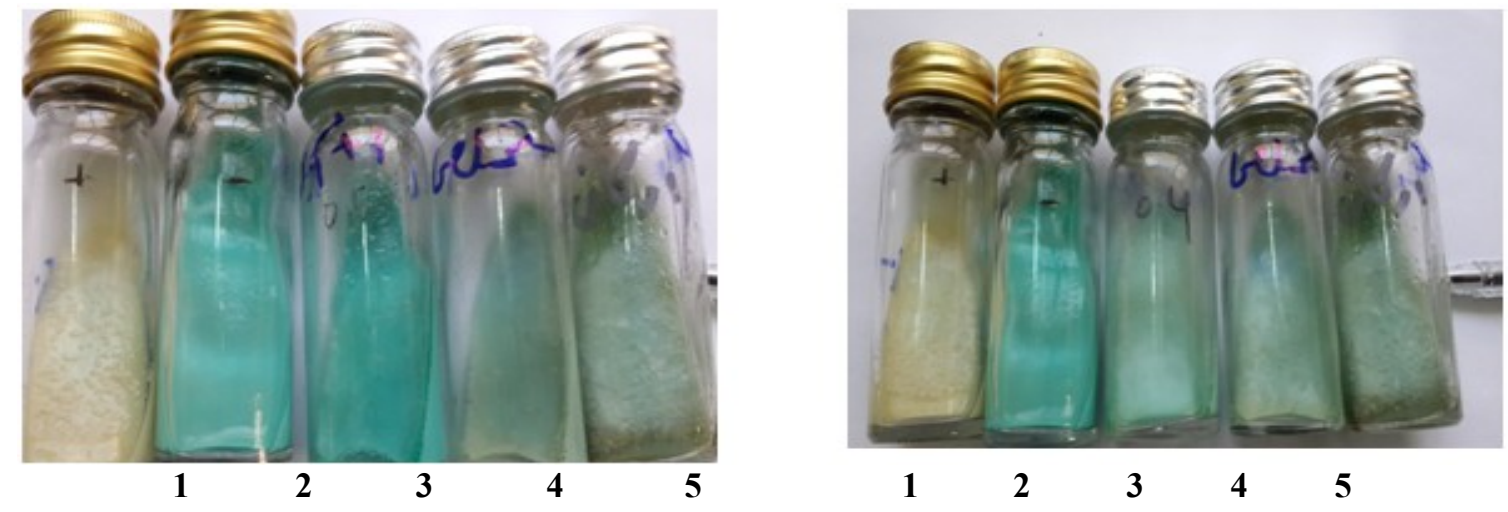

C:D:

1.Positive control tube.1. Positive control tube.

2.Negative control tube.2.Negative control tube.

3. L-J with the compound (9.5:0.5) $\mathrm{ml}$. 3. L-Jwith compound(9.6:0.4) $\mathrm{ml}$.

4.L-J with sidr extract only.4. L-J withsidr extract only.

5. L-J withArgan oil only. 5. L-J withArgan oil only.
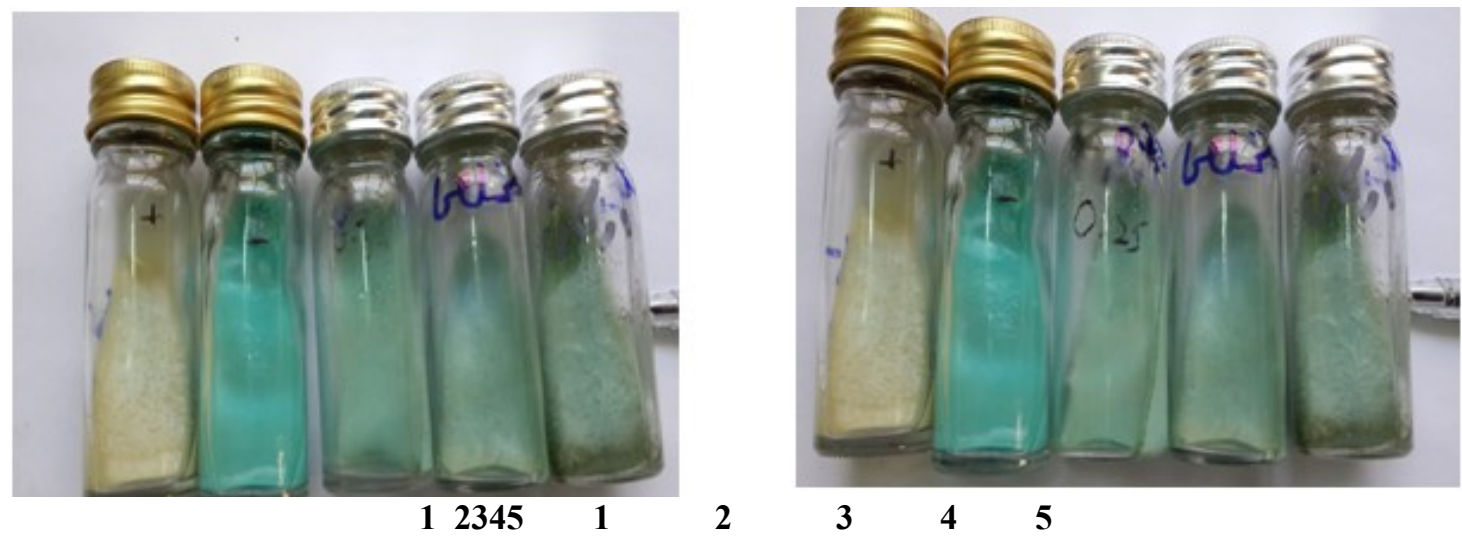

E:

F:

2

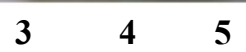

1.Positive control tube.1.Positive control tube.

2.Negative control tube.2. Negative control tube.

3. L-J with the compound (9.7:0.3) $\mathrm{ml}$. 3. L.J. medium(9.75:0.25) $\mathrm{ml}$

4. L-J with sidr extract only.4.L-J with sidr extract only.

5. L-J with Argan oil only. 5. L-J with Argan oil only. 

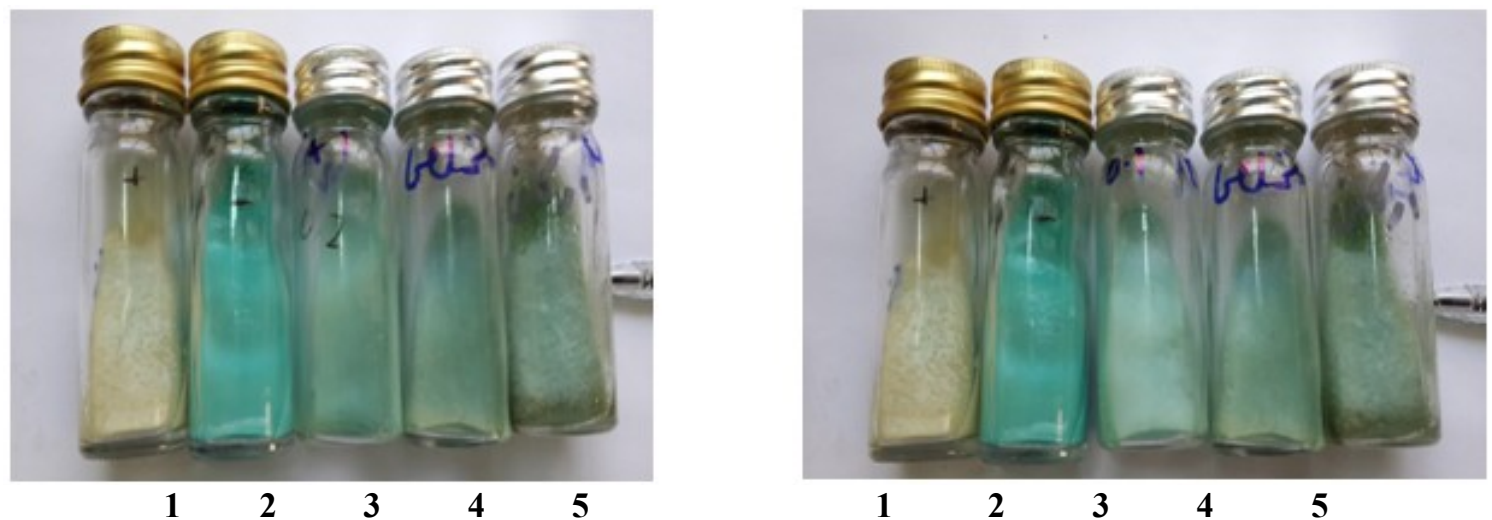

G: H:

1. Positive control tube. 1. Positive control tube.

2.Negative control tube. 2. Negative control tube.

3. L-J with the compound (9.8: 0.2) $\mathrm{ml}$. 3. L.Jwith the compound (9.9: 0.1) $\mathrm{ml}$.

4. L-J with sidr extract only. 4. L-J with sidr extract only.

5. L-J with Argan oil only. 5. L-J with Argan oil only.

Figure 1, (A-H) illustrate the results combined compound on TB-bacilli.

\section{Conclusion}

This study settles the activity of mixture (Argan oil $100 \%$ and Sidr extract $62.5 \mathrm{~g} \backslash \mathrm{L}$ ) as anti- TB agent.The novel compound used in this study revealed valuable effect against TB. Afterincorporation with culture media.

\section{Acknowledgements}

We would like to thankful every person who had helped us in this Research.

\section{References}

[1]. RBrosch, SV.Gordon,A.Pym,K.Eiglmeier,T. Garnier, andS.T. Cole, Comparative genomics of the mycobacteria. Int J MedMicrobiol; 290(2), 2000, 143-152.

[2]. S.Mostowy, D. Cousins, J. Brinkman, A.Aranaz, and M.A. Behr, Genomic deletions suggest a phylogeny for the Mycobacteriumtuberculosis complex. J Infect Dis; 186(1), 2002, 74-80.

[3]. N.W.Schluger, The pathogenesis of tuberculosis: the first one hundred (and twenty-three) years. Am J Respir Cell MolBiol; 32(4), 2005, 251-256.

[4]. C.J.Gallant,S. Malik,N.Jabado,M .Cellier,L.Simkin,B. B.Finlay, E. A.Graviss,P.Gros,J. M .Musser, and E.Schurr, Reduced in vitro functional activity of human NRAMP1 (SLC11A1) allele that predisposes to increasedrisk of pediatric tuberculosis disease, Genes Immun. 8(8), 2007, 691-8.

[5]. A.Wright, M.Zignol, andA. Van Deun, Epidemiology and ant tuberculosis drug resistance 2002-2007: an updated analysis of the global project on anti-tuberculosis drug resistance surveillance,Lancet 373, 2009, 1861-1873.

[6]. World Health Organization, WHO report 2011,Global tuberculosis control,( WHO/HTM/TB/Geneva: WHO, 2011).

[7]. MC.Raviglione, Reichmann and Hershfield's tuberculosis: A Comprehensive; International Approach. 3 ed, ( New York: Informa Healthcare USA, 2006).

[8]. $\quad$ "Biosphere Reserve Information",UNESCO.Archived from the original on 18 September 2007.Retrieved 2007.

[9]. F. Khallouki,C.Younos,R.Soulimani,T.Oster,Z. Charrouf, B .Spiegelhalder,H.Bartsch, andR.W .Owen, "Consumption of argan oil (Morocco) with its unique profile of fatty acids, tocopherols, squalene, sterols and phenolic compounds should confer valuable cancer chemopreventiveeffects",European journal of cancer prevention. 12 (1), 2003, 67-75.

[10]. Z.Charrouf, and D .Guillaume, "Argan oil: Occurrence, composition and impact on human health", European Journal of Lipid Science andTechnology.110 (7), 2008, 632-635.

[11]. H.E.Monfalouti,D .Guillaume,C.Denhez, andZ.Charrouf, Therapeutic potential of Argan oil: a review, JPharmPharmacol. Dec, 62(12), 2010, 1669-75.

[12]. Z. Charrouf, and D. Guillaume, "Phenols and Polyphenols from Arganiaspinosa", Am. J. of Food Technology 2 (7), 2007, 679683.

[13]. H. E.Yossef,A.A.Khedr, and M.Z. Mahran, Hepatoprotective activity and antioxidant effects of El Nabka (Zizphusspina-christi) fruits on rats hepatotoxicity induced by carbon tetrachloride, Nat. Sci. 9, 2011, 1-7.

[14]. M.E. Abalaka, S.Y. Daniyan, andA. Mann, Evaluation of the antimicrobial activities of two Ziziphusspecies (ZiziphusmauritianaL. and Ziziphusspina-christiL) on some microbial pathogens, Afr. J. Pharm. Pharmacol. 4, 2010, 135-139 .

[15]. J. Asgarpanah, andE.Haghighat, Photochemistry and pharmacologic properties ofZiziphusspinachristi (L.)Willd.African Journal ofpharmacy and Pharmacology Vol. 6, 2012, 2332-2339. 
[16]. K.C. Hasan, and A. K . AL-saffar, Anewly Invented chemical Disinfectant can eradicate colonic Bacterial contamination of the Skin, International Journal of Medicine and Pharmaceutical Sciences (IJMPS). 4(2), 2014, 47-54.

[17]. J. Dhari, N. Mohammed, andP. Mehdi, Evaluate the effectiveness of the antibacterial activity of alcoholic and aqueous extracts of leaves of Sidr, Basrah Journal of Science (B). 25(1), 2007, 1-16 (the article was published in Aarabic language).

[18]. W. Winn, S. Allen, W. Janda, E.Koneman, G.Procop, P.Schreckenberger, and G. Woods, Koneman's color atlas and textbook of diagnostic microbiology, (6th edition.Lippincott Williams \& Wilkins. 2006). 